\title{
Letter to the Editor regarding "Clinical behaviors and prognoses of high- and low-risk parotid malignancies based on histology" by Tao L. et al.
}

\author{
Dominik Stodulski $^{1}$ (D) Bogusław Mikaszewski ${ }^{1} \cdot$ Jerzy Kuczkowski $^{1}$
}

Received: 15 March 2019 / Accepted: 25 March 2019 / Published online: 29 March 2019

(c) Springer-Verlag GmbH Germany, part of Springer Nature 2019

\section{Dear Editor,}

We read with interest the study of Tao L. et al. entitled "Clinical behavior and prognoses of high- and low-risk parotid malignancies based on histology". The authors showed significant differences within parotid tumors depending on their grade (high-risk vs. low-risk histology). High-risk malignancies are characterized by worse overall and disease-free survival, and more frequently occur with perineural invasion, pain, facial nerve palsy, lymphatic metastases and recurrence [1]. These results confirm our own observations that the most important prognostic factors are the $\mathrm{T}$ - and $\mathrm{N}$-stages and the tumor grade [2]. However, we must pay attention to several elements in this work that raise our doubts.

The histology of tumors and risk stratification presented in the paper are based on the obsolete WHO histological classification of salivary gland tumors from 2005, but not on currently applicable from 2017 (e.g., polymorphous lowgrade adenocarcinoma at present polymorphous adenocarcinoma) [3].

The principles of risk stratification come from the work of Seethal (2009) and raise certain doubts, e.g., metastasizing pleomorphic adenoma is classified as high risk although it is an extremely rare situation (and removed from the latest WHO classification) of distant metastases after the surgical treatment of histologically benign pleomorphic adenoma [4].

In our opinion, the basis for stratification of the material should be histological revision/reclassification of diagnoses with the determination of tumor grade, especially since 7 of the 65 tumors in this series could not be stratified.

This comment refers to the article available at: https://doi. org/10.1007/s00405-018-5224-9.

Dominik Stodulski

dstodulski@gumed.edu.pl

1 Department of Otolaryngology, Medical University of Gdansk, ul. Smoluchowskiego 17, 80-214 Gdańsk, Poland
Surprising also is such a large proportion of polymorphous adenocarcinoma in the parotid gland 11/65 (17\%), which is typical for small salivary glands and in major glands is extremely rare!

In addition, epithelial and non-epithelial parotid tumors were mixed in this study. Lymphomas are treated systemically, not surgically (the procedure is a kind of open biopsy). The results of treatment of lymphomas together with carcinomas cannot be presented. What do the "other types" in the pathology type mean?

I hope the authors will be able to clarify our doubts.

Funding None.

\section{Compliance with ethical standards}

Conflict of interest The authors declare that they have no conflict of interest.

Ethical approval For this type of study formal consent is not required.

\section{References}

1. Tao L, Zhang D, Zhang M, Zhou L (2019) Clinical behaviours and prognoses of high- and low-risk parotid malignancies based on histology. Eur Arch Otorhinolaryngol 276(2):497-503. https ://doi.org/10.1007/s00405-018-5224-9 (Epub 2018 Dec 1)

2. Stodulski D, Mikaszewski B, Stankiewicz C (2012) Are all prognostic factors in parotid gland carcinoma well recognized? Eur Arch Otorhinolaryngol 269:1019. https://doi.org/10.1007/s0040 5-011-1716-6

3. El-Naggar AK, Chan JKC, Grandis JR, Takata T, Slootweg PJ (eds) (2017) WHO classification of head and neck tumours, 4th edn. IARC, Lyon

4. RR Seethala (2009) An update on grading of salivary gland carcinomas. Head Neck Pathol 3(1):69-77

Publisher's Note Springer Nature remains neutral with regard to jurisdictional claims in published maps and institutional affiliations. 